\title{
Breast conservation in breast cancer patients with cardiac pacing devices
}

\author{
Mark Trombetta ${ }^{1,3,4}$, Yongbok Kim ${ }^{1,3,4}$, Thomas B. Julian ${ }^{2,3,4}$ \\ 1. Department of Radiation Oncology, Allegheny General Hospital, 320 East North Avenue, Pittsburgh, Pennsylvania, USA. \\ 2. Department of Surgery, Allegheny General Hospital, Pittsburgh, Pennsylvania, USA. 3. Temple University School of \\ Medicine, Pittsburgh Campus, Pittsburgh, Pennsylvania, USA. 4. Drexel University College of Medicine, Allegheny Campus, \\ Pittsburgh, Pennsylvania, USA
}

Correspondence: Mark Trombetta. Address: Department of Radiation Oncology, Allegheny General Hospital, 320 East North Ave. Pittsburgh, PA 15212, USA. E-mail: mtrombet@wpahs.org

Received: May 3, 2012

Accepted: J une 26, 2012

Online Published: November 7, 2012

DOI : $10.5430 /$ jst.v2n6p1

URL: http://dx.doi.org/10.5430/jst.v2n6p1

\section{Keywords}

Radiation, Pacemaker, Defibrillator, Accelerated partial breast radiotherapy, Breast, Lumpectomy

\section{Introduction}

Patients that have implantable pacemakers and defibrillators who develop localized breast cancer have historically been considered better candidates for mastectomy than breast conservation therapy (BCT) due to the risk of device malfunction. Many of these patients are desirous of BCT but have been felt to be unsuitable due to the disruptive potential to the pacing device resulting from the primary radiation beam and electromagnetic fields that occur during delivery of megavoltage irradiation. The use of pacing devices is growing rapidly and more patients with early-stage breast cancers have implanted devices. Newer treatment techniques involving the use of accelerated partial breast radiotherapy (APBI) ${ }^{[1,2]}$ accompanied by recently published guidelines detailing suitability criteria for APBI ${ }^{[3]}$ have changed the breast cancer paradigm. We present the Allegheny General Hospital experience in patients with early-stage breast cancer and implanted cardiac pacing devices who desired breast preservation.

\section{The need for a continued persistent focus on breast preservation}

Breast conservation therapy in early-stage breast cancer has a long history with two major studies reporting equivalence in $20+$ year experiences in safety, local control, cosmesis, overall survival, and patient preference ${ }^{[4,5]}$. Researchers have reported improvement in self-esteem and body self-image when the breast is preserved using breast conservation techniques ${ }^{[7,8,9]}$. With the current trends devolving away from mastectomy, we believe the presence of cardiac pacing devices should have little impact the choice of breast preservation, provided that safety and quality criteria can be accommodated. In 2004, Solan et al, outlined safety criteria standards for patients with cardiac pacing devices who require radiotherapy ${ }^{[10]}$. However, the current generation of cardiac pacing devices are less radiotolerant, with most manufacturers defaulting to a 200 cGy maximal limit or an ALARA (as low as reasonably achievable) dose recommendation. The complexity of these devices, including the institution of complementary metal-oxide semiconductor (CMOS) technology, may make them at least 10-fold less tolerant to radiotherapy than just 15-20 years ago. While these statements suggest an argument toward lesser use of radiotherapy, we argue for continued focus on breast preserving therapy when possible. Allegheny General Hospital radiation oncologists and breast surgeons have successfully treated a number of patients with 
a pacemaker either ipsilateral or contralateral to their breast cancer. Only a single incident of a non-life threatening pacemaker reset to default has occurred and this was easily corrected by the manufacturer's representative. Treatment has been delivered using both whole breast radiotherapy (WBRT) (3 patients), and APBI with both high dose rate brachytherapy (HDR) and three-dimensional conformal radiotherapy (3D-CRT) (13 patients). The WBRT and APBI patients were treated in accordance with the National Surgical Breast and Bowel Project (NSABP) protocol B-39/Radiation Therapy Oncology Group (RTOG) 0413 guidelines ${ }^{[11]}$; although the WBRT patients required a shortening of the superior border of the tangent fields by $1.5-2.0 \mathrm{~cm}$ to protect the device. Despite lowering the superior border, two of three WBRT patients met the protocol planning volumes for APBI. As APBI is an evolving standard of care with two studies demonstrating $10+$ year equivalence data in local control compared to WBRT ${ }^{[1,2]}$, we were comfortable with the border reduction since these patients were highly desirous of BCT. The single patient who did not fit the protocol required only a $0.5 \mathrm{~cm}$ reduction at the superior border of the treatment bed. Because the relatively large planning margins required are due in large part to respiratory motion ( $2.5 \mathrm{~cm}$ total), and chest wall motion in this region is physiologically limited, we felt this acceptable despite the usual motion concerns as the superior aspect of the thoracic cavity (and therefore the breast) demonstrates the least variation which may require less planning target volume margin.

We employ an in-house pacemaker protocol, that includes a graphic instructive chart (Table 1), which is based on the Solan recommendations ${ }^{[10]}$ and have established minimal distance guidelines (target volume to device) based on ex-vivo computerized simulation for both external radiotherapy and HDR. The device manufacturer is contacted to obtain the device specific radiation tolerances and virtual pre-plans are performed to estimate the maximal dose to the device. Once the patient has been approved for treatment following verification of safe dose limit predictions, the device is monitored during two consecutive treatments by thermoluminescent or electronic dosimetry. All patients met the maximal dose standards both virtually and real-time and the tumor bed coverage averaged $98 \%$.

Table 1. Minimum Recommended Pacemaker Interventions

\begin{tabular}{lllll}
\hline & $\begin{array}{l}\text { Cardiologist } \\
\text { Consult before } \\
\text { XRT }\end{array}$ & EKG Monitor & $\begin{array}{l}\text { Vital Signs Pre and } \\
\text { Post XRT }\end{array}$ & Dosimeter measurement first two fractions \\
\hline $\begin{array}{l}\text { Dependant High } \\
\text { Risk }\end{array}$ & Yes & first 3 tx's or & daily & Yes \\
$\begin{array}{l}\text { Dependant Low } \\
\text { Risk }\end{array}$ & Yes & first 3 tx's & first 3 tx's & Yes \\
$\begin{array}{l}\text { Non-Dependant } \\
\text { High Risk }\end{array}$ & Yes & No & first 3 tx's & Yes \\
$\begin{array}{l}\text { Non-Dependant } \\
\text { Low Risk }\end{array}$ & Yes & No & first 3 tx's & Yes \\
\hline
\end{tabular}

* AICD = Automatic Implantable Cardiac Defibrillator

High risk = pacer constant use necessary for life or AICD

Dependant High Risk: Dependant Pacemaker or AICD that is receiving $\geq 2 \mathrm{~Gy}$ and/ or $\leq 10 \mathrm{~cm}$ from field edge.

Dependant Low Risk: Dependant Pacemaker or AICD that is receiving $<2 \mathrm{~Gy}$ and $>10 \mathrm{~cm}$ from field edge.

Non-Dependant High Risk: Non-Dependant Pacemaker or AICD that is receiving $\geq 2 \mathrm{~Gy}$ and/ or $\leq 10 \mathrm{~cm}$ from field edge.

Non-Dependant Low Risk: Non-Dependant Pacemaker or AICD that is receiving $<2 \mathrm{~Gy}$ and $>10 \mathrm{~cm}$ from field edge.

\section{Conclusions}

BCT is feasible in patients who prefer BCT when presenting with early stage breast cancers and who also have implanted cardiac devices in the contralateral or ipsilateral breast as long as both the limits of cardiac device tolerance and target volume coverage can be achieved. Breast conservation should be considered for these patients who meet appropriate APBI criteria. 


\section{References}

[1] Polgar C, Major T, Fodor J, et al. Accelerated partial-breast irradiation using high-dose-rate interstitial brachytherapy: 12ᄀyear update of a prospective clinical study. Radiother Oncol. 2010; 94:274-9. PMid:20181401

[2] Antonucci J V, Wallace M, Goldstein N S, et al: Differences in patterns of failure in patients treated with accelerated partial breast irradiation versus whole-breast irradiation: A matched pair analysis with 10 year follow-up. Int J Radiat Oncol Biol Phys. 2009 Jun 1; 74 (2): 447-52. PMid:19058921 http://dx.doi.org/10.1016/j.ijrobp.2008.08.025

[3] Smith B, Arthur D W, Buchholz T, et al: Accelerated partial breast consensus statement from the American Society for Radiation Oncology. Int J Radiat Oncol Bio Phys. 2009 Jul 15; 74 (4): 987-1001. PMid:19545784 http://dx.doi.org/10.1016/j.ijrobp.2009.02.031

[4] Fisher B, Anderson S, Bryant J, et al: Twenty-year follow-up of a randomized trial comparing total mastectomy, lumpectomy, and lumpectomy plus irradiation for the treatment of invasive breast cancer. N Eng J Med. 2002; 347: 1233-41. PMid:12393820 http://dx.doi.org/10.1056/NEJMoa022152

[5] Veronesi U, Cascinelli N, Mariani L, et al: Twenty-year follow-up of a randomized study comparing breast-conserving surgery with radical mastectomy for early breast cancer. N Engl J Med. 2002; 347: 1227-32. PMid:12393819 http://dx.doi.org/10.1056/NEJMoa020989

[6] Fowble BL, Solin LJ, Schultz DJ, et al: Ten year results of conservative surgery and irradiation for stage I and II breast cancer. Int J Radiat Oncol Biol Phys. 1991; 21: 269-277. http://dx.doi.org/10.1016/0360-3016(91)90771-U

[7] Ganz PA, Schag CCA, Lee JJ, et al: Breast conservation versus mastectomy. Is there a difference in psy $\neg$ chological adjustment or quality of life in the year after surgery? Cancer. 1992; 69: 1729-38. http://dx.doi.org/10.1002/1097-0142(19920401)69:7<1729::AID-CNCR2820690714>3.0.CO;2-D

[8] Rowland J, Desmond, K, Meyerowitz B, et al: Role of Breast Reconstruction in Physical and Emotional Outcomes among Breast Cancer Survivors. JNCI. 2000; 92: 1422-1429. PMid:10974078 http://dx.doi.org/10.1093/jnci/92.17.1422

[9] Maunsell E, Brisson. J, Deschhes. L: Psychological Distress after Initial Treatment for Breast Cancer: A Comparison of Partial and Total Mastectomy. J Clin Epidemiol. 1989; 42: 765-771. http://dx.doi.org/10.1016/0895-4356(89)90074-7

[10] Solan AN, Solan MJ, Bednarz G, et al: Treatment of patients with cardiac pacemakers and implantable cardioverter-defibrillators during radiotherapy. Int J Radiat Oncol Biol Phys. 2004; 59: 897-904. PMid:15183493 http://dx.doi.org/10.1016/j.ijrobp.2004.02.038

[11] Vicini FA, White J, Arthur D, et al: NSABP B-39/RTOG 0413 protocol, A randomized phase III study of conventional whole breast irradiation (WBI) versus partial breast irradiation (PBI) for women with stage 0 , I or II breast cancer [Internet]. Available from: http://www.rtog.org. 